\title{
Estimación de la eficiencia de la Bolsa de Valores de Lima (1990-2018)1
}

\section{Estimation of the efficiency of the Lima Stock Exchange (1990-2018)}

\author{
David Gonzalo Chávez Vargas ${ }^{2}$ \\ Superintendencia Nacional de Aduanas y de Administración Tributaria \\ dchavezv@sunat.gob.pe
}

Recibido: 25/03/2021 - Aceptado: 18/05/2021 - Publicado: 06/12/2021

\section{RESUMEN}

El presente artículo presenta la aplicación de un modelo VAR restringido a la relación entre el rendimiento de los activos transados en la Bolsa de Valores y los fundamentos económicos agregados, estos son: consumo agregado, exportaciones, inversión agregada, tipo de cambio real. Utilizando datos trimestrales se concluye que existe eficiencia semi-fuerte relacionada con los fundamentos señalados lo que es indicador del cumplimiento de las funciones del sistema financiero en la economía nacional.

Palabras Clave: Bolsa de Valores, Eficiencia de Mercado, Modelo VAR, Mercados Emergentes.

Clasificación JEL: G14.

\section{ABSTRACT}

This paper shows the estimation of a restricted VAR model at the relation between the return of assets traded at Bolsa de Valores de Lima (Lima stock Exchange) and the economic fundamentals: aggregate consumption, exports, aggregate investment and real exchange rate. Using quarterly data we conclude that exists semi-strong efficiency in relation to the fundamentals, this could be an indicator of achievment of the functions of financial system in the domestic economy.

Key Words: Stock Market, Market Efficiency, VAR Model, Emerging Markets. JEL classification: G14.

(C) Los autores. Este artículo es publicado por Pensamiento Crítico de la Facultad de Ciencias Económicas, Universidad Nacional Mayor de San Marcos. Este es un artículo de acceso abierto, distribuido bajo los términos de la licencia Creative Commons Atribucion - No Comercia_Compartir Igual 4.0 Internacional. (http://creativecommons.org/licenses/by-nc-sa/4.0/) que permite el uso no comercial, distribución y reproducción en cualquier medio, siempre que la obra original sea debidamente citada. 


\section{Introducción}

El mercado de capitales como intermediario en la asignación de recursos ha sido un tema de amplio debate en la teoría económica, ya que estos deben canalizar de manera eficiente los recursos de los agentes con superávit de recursos a otros que los requieren para fines de adquisición o expansión de capital. La eficiencia se suele aproximar con dos métricas principales: la velocidad de ajuste de los precios de las acciones que transan en el mercado y, los costos relativos por participar en el mercado.

\section{Marco Teórico}

Uno de los pioneros en analizar la hipótesis de eficiencia es Fama (1970) donde además de hacer una exploración teórica de los mercados eficientes, hace un análisis empírico de los security prices, la hipótesis de la eficiencia se plantea como sigue:

"La mayor parte del trabajo disponible se basa solo en el supuesto de que las condiciones de equilibrio del mercado pueden expresarse (de alguna manera) en términos de retornos esperados. En términos generales, al igual que los dos modelos de parámetros, tales teorías postularían que, dependiendo de algún conjunto de información relevante, el rendimiento esperado de equilibrio de un valor es una función de su "riesgo"." (Fama 1970, p. 384)

Como se puede ver en el análisis de los precios, su representación mediante retornos juega un papel importante. Al hacer esta discusión se adentra en analizar tres modelos sobre los retornos:

1. Retorno esperado o modelos de "juego justo".

2. El modelo de submartingale.

3. El modelo de paseo aleatorio.

Concluyendo de esto, que los modelos de paseo aleatorio son más eficientes que los de retorno esperado, pues en los modelos de paseo aleatorio los gustos de los inversores evolucionan con la nueva información que se va previendo. 
Fama (1970) señala que el rol primario del mercado de capitales es la asignación de la propiedad del stock de capital de la economía. En términos "ideales" es un mercado en el cual los precios ofrecen señales precisas para la asignación de recursos: las firmas hacen decisiones de producción-inversión, y los inversores pueden escoger entre los valores que representan esas actividades de las empresas bajo el supuesto que los precios "reflejen completamente" toda la información, tal mercado es llamado eficiente. En el contexto de la tradición de Markowitz y el modelo CAPM inicial, tal mercado debe ser diversificado para que permita al participante tomar una canasta de acciones de tamaño suficiente como para que sus objetivos de rentabilidad-riesgo se cumplan en el plazo de inversión relevante.

Luego de esto se puede pasar a la evidencia empírica, en los diversos estudios se puede ver que la influencia de los mercados eficientes puede darse en tres test: a) los test de forma débil (con el conjunto de información de precios), b) Semi-fuerte (es el conjunto de información más información pública) c) Fuerte donde la información es dirigida por llamados insiders.

Como Fama dijo en 1970, los costos de transacción implican una gran parte de que la hipótesis de eficiencia no se cumple, el hecho que las empresas estén o no muy informadas hacen que la hipótesis de eficiencia tienda a no cumplirse.

El artículo de Fama (1991) es recuento de la hipótesis de eficiencia luego de 20 años donde la literatura se ha extendido. Fama explica la hipótesis de eficiencia como:

"Considero que la hipótesis de eficiencia del mercado es la simple declaración de que los precios de activos reflejan completamente toda la información disponible." (Fama 1991, p. 1575)

La revisión que hace entorno a las áreas de la hipótesis de eficiencia como la débil, semi-fuerte y fuerte los respectivos test que se utilizan. Para este caso nos interesan los test, tales como el test de volatilidad, donde los rendimientos esperados y la variación de precios en acciones pueden ser dirigidos por dividendos o por otras variables como la tasa de interés o variables de estructura. Resulta interesante la revisión que 
se hace de los trabajos pioneros que sustentan el modelo de analizar esta hipótesis con modelos de volatilidad.

El otro modo para ver la hipótesis de eficiencia es con rendimiento de retorno transversal, referida principalmente a los modelos "asset-pricing" que en sí mismo no son pruebas, sino que ya suponen esta eficiencia como una hipótesis previa, se sostiene que estas pruebas son eficientes en el sentido que relacionan la eficiencia con el modelo propuesto de valoración de activos.

Summers (1986) reseña algunos métodos aplicados a la medición de la eficiencia. Señala la dificultad asociada a que la potencia de los tests de medición tiene menos potencia cuando "más largo" es el horizonte de inversión de los agentes que organiza. Medidas como la valoración de mercado puede diferir fuertemente de la valoración bajo un marco de expectativas racionales, asimismo se cuestiona el papel de la especulación para "asegurar" valorizaciones racionales, subsistiendo los problemas de identificación de las fuentes de valorización en estos mercados.

Poterba y Summers (1989) analizan la evidencia estadística en relación a los componentes transitorios de la fracción de la varianza en los retornos de los valores accionarios. Los autores realizan una simulación de retornos de 25,000 secuencias de 720 pares de retornos bajo un proceso generador de datos con varianza determinada; mediante este análisis de los ratios de la distribución de los retornos se identifica la fuente de retornos superiores, asumiendo una varianza decreciente para algunos valores seleccionados. El artículo demuestra que los ratios de la varianza están entre los más potentes para detectar la reversión a la media de los precios de las acciones. En la aplicación de los test de ratios de varianzas a los retornos de mercado de Estados Unidos (1871-1986) encuentran evidencia consistente que los retornos muestran evidencia de eficiencia en el mercado.

En Fama (1998), extenderá un más la idea de mercados eficientes extendiéndola a los rendimientos a largo plazo, pues las anomalías que se dan en el largo plazo aparentemente a los métodos de medición, pues cambiando estos se puede obtener interesantes resultados. Así comienza haciendo una división de estas anomalías en reacción insuficiente y reacción exagerada. El problema fundamental surge del uso de los malos modelos pues se cómo se analizó anteriormente la hipótesis de eficiencia 
viene ligada al estudio de los rendimientos promedio, estos vienen a ser informaciones incompletas, pues como son rendimientos promedio tiene a subestimarse o sobreestimarse información que de los patrones.

Demirgüç-Kunt y Levine (1993) estudian el papel de los mercados de valores en los países en desarrollo, señalando que hay posiciones contrapuestas en cuanto al papel de los mercados de valores, por un lado existe un sector crítico que afirma que estos mercados son altamente especulativos, y que los precios no pueden ser explicados por sus fundamentos económicos, a raíz de la pobre información que proveen a sus participantes, altos costos de transacción, regulación inefectiva e incluso insuficiente competencia.

La Porta, R., Lakonishok, J, Shleifer, A. y Vishny, R. (1997) examinan la hipótesis de que el retorno superior de los llamados "value stocks" es el resultado de errores de los inversionistas, demostrando de esta manera la ineficiencia para predecir los retornos por parte de los analistas del mercado. Los autores estudian las reacciones de los precios de las acciones alrededor de los anuncios de ganancias para acciones "value stock" versus acciones de empresas grandes en un periodo de cinco años. Mediante un test de medias (t-test), los autores comparan los retornos de diferentes niveles de relación (Valor en Libros/Valor de Mercado), bajo diferentes niveles de intervalos de tiempo (de 1 hasta 20 trimestres).

Ojah y Karemera (1999), prueban la hipótesis de eficiencia y los precios de las acciones, su método es estadístico, en el cual utilizan pruebas de promedios móviles y pruebas de varianza demostrando que los principales mercados de Latinoamérica tienen una forma de caminata aleatoria y siguen una forma débil de hipótesis de eficiencia.

De la Torre, Gozzi y Schmukler (2007) estudian los efectos del desarrollo del mercado de valores en el marco de los procesos de liberalización de las economías de los años noventa, mediante un análisis empírico encuentran que las reformas implementadas han incrementado la capitalización, el intercambio de valores, y el levantamiento de capital de las empresas participantes. Pero estos desarrollos también propician la internacionalización de las empresas participantes (las de mayor tamaño), pudiendo disminuir la liquidez del mercado, lo cual a su vez disminuye el acceso al mercado de valores a las medianas empresas. 
Iqbal, Nawaz y Umer (2017) utilizan un modelo autorregresivo de retardos distribuidos para investigar el impacto de las principales variables macroeconómicas sobre el valor de las acciones en Pakistán, encontrado evidencia de cointegración entre el índice de la bolsa y los fundamentos macroeconómicos del país. Los resultados se muestran para el corto y el largo plazo, en el caso del modelo de largo plazo encuentran asociación positiva entre la oferta de dinero y los rendimientos de los activos, en el caso del tipo de cambio se encuentra una relación negativa, en línea con el efecto sugerido en las teorías corrientes del tipo de cambio. Cabe mencionar que para una especificación de largo plazo se encuentra que la depreciación de la moneda está asociada con un menor rendimiento del mercado accionario.

Karki (2018) estudia igualmente los factores macroeconómicos determinantes del performance del mercado de acciones en Nepal, considerando el periodo 1994-2016 con datos anuales se estima un modelo de largo plazo, realizando las pruebas usuales de raíz unitaria y cointegración; usando las variables agregadas principales: inflación, tasa de interés de corto plazo y un indicador de oferta convencional (M2), encuentra influencia de estas variables en el índice accionario, sin embargo no observan evidencia de cointegración entre los indicadores macroeconómicos y el índice de la bolsa.

Un mercado de valores que funcione adecuadamente propicia, entre otros a) un crecimiento del ahorro nacional, b) una asignación eficiente de la inversión. El primer rol se lleva a cabo proveyendo al inversionista un instrumento adicional que refleje sus preferencias de riesgo y liquidez. El segundo rol tiene una connotación dinámica más amplia, las firmas obtienen el ahorro de los agentes superavitarios, pero los resultados (en términos de beneficios) se observan varios periodos después de la decisión de inversión, en el transcurso de ese tiempo se agrega información relevante que el inversionista puede evaluar para cambiar su decisión de inversión si lo considera rentable, o le permite minimizar alguna pérdida asumida.

La disponibilidad de datos financieros en las últimas décadas, ha mejorado la viabilidad de medir y evaluar indicadores de desarrollo del mercado de capitales, asimismo el importante desarrollo de métodos econométricos de la última década también ha posibilitado analizar un mayor número de fenómenos económicos, en particular las técnicas asociadas a 
las series de tiempo han mostrado una amplia expansión, una de las cuales se utiliza en el presente documento.

\section{Metodología}

Tomando como base el trabajo de Leigh (1997) que tiene como objetivo examinar la eficiencia de la bolsa de Singapur adaptamos esta medología para el caso peruano. Para ello usa un rango de métodos (test de causalidad de Granger, modelos de series temporales). En una primera etapa se lleva a cabo los test de raíz unitaria y la ecuación de cointegración. Bajo el enfoque de Johansen, evaluando si los betas de la ecuación son significativos Se plantea un modelo de variables cointegradas para determinar la eficiencia del mercado.

En el presente punto describimos las hipótesis del trabajo, estas ponen en términos de afirmaciones sujetas a comprobación empírica nuestra idea principal, la bolsa de valores nacional es eficiente en sentido semi fuerte en el periodo de estudio.

Para ello se analiza los coeficientes $\mathrm{a}_{\mathrm{ij}}$ de la matriz del modelo VAR estructural que asocia los retornos del mercado con los fundamentos económicos del país. En la siguiente matriz:

$$
\left(\begin{array}{c}
r m_{t} \\
c_{t} \\
i_{t} \\
x_{t} \\
t c r_{t}
\end{array}\right)=\left(\begin{array}{llllll}
a_{11} & a_{12} & a_{13} & a_{14} & a_{15} & a_{16} \\
a_{21} & a_{22} & a_{23} & a_{24} & a_{25} & a_{26} \\
a_{31} & a_{32} & a_{33} & a_{34} & a_{35} & a_{36} \\
a_{41} & a_{42} & a_{43} & a_{44} & a_{36} & a_{46} \\
a_{51} & a_{52} & a_{53} & a_{54} & a_{55} & a_{56}
\end{array}\right)\left(\begin{array}{c}
r m_{t-i} \\
c_{t-i} \\
i_{t-i} \\
x_{t-i} \\
t c r_{t-i}
\end{array}\right)
$$

$\mathrm{r}_{\mathrm{m}}$ son los rendimientos del mercado, c es el agregado de consumo de la economía, i es el agregado inversión, x es el agregado de exportaciones y tcr es el tipo de cambio real de la economía.

El número de vectores autorregresivos significativos (y su signo) indicará que los rendimientos del mercado de valores están fundamentados en los agregados económicos, siendo además los elementos aij, los factores de influencia del sistema.

Podemos indicar entonces que: 
H0 : número de vectores autoregresivos significativos $=5$.

Ha : número de vectores autoregresivos significativos $<5$.

Los elementos $\mathrm{a}_{\mathrm{ij}}$ de la matriz del sistema de ecuaciones anterior son significativos, en general se espera que los elementos sean menores a uno, y que sea significativo al nivel de confianza escogido.

Cabe señalar que su utiliza un VAR restringido en el caso se necesite probar una relación estrucutural, la cual se debe plantear en este modelo tal que exprese una posible relación con significado económico, esto es se incorpora explícitamente posibles relaciones de largo plazo que deberían mantener las variables entre sí.

Se eligió el periodo 1990-2018 por corresponder a una etapa posterior a la ejecución del programa de estabilización de la economía, constituyendo el periodo 28 años posteriores al shock, periodo de interés para un estudio de largo plazo de la dinámica de nuestro sector financiero.

$\mathrm{Rt}=$ los retornos del mercado de valores de la Bolsa de Valores del Perú en el periodo 1992-2015.

PBI = nivel de producción agregado de la economía, medida en millones de nuevos soles reales al año 2007.

Tasa de interés = Tasa de referencia del BCRP, en el mismo periodo base.

Nivel de precios = Índice de Precios al Consumidor en Lima Metropolitana. Inversión = Componente de inversión, en el desagregado del PBI por el método del gasto, medida en millones de nuevos soles reales al año 2007.

Tipo de cambio real $=$ Cotización spot de fin de periodo del tipo de cambio dólar estadounidense / Sol * (Índice de precios extranjero/ Índice de precios doméstico).

Los datos a analizar se recogerán de los servicios de información disponibles, estos son bases de datos de servicios como Economática, así como los indicadores disponibles en la página web de la Bolsa de Valores de Lima. Los indicadores macroeconómicos se recogerán de la página web del Banco Central de Reserva del Perú. 
Los retornos de la BVL corresponden a los retornos de los componentes de la BVL actualizados año a año por la mencionada institución, en este sentido es un indicador usado por los inversionistas como un indicador general de la evolución del mercado.

En función del tiempo, la muestra recogida está limitada por el desarrollo del mercado de valores, que es de reciente desarrollo en el mercado financiero nacional en comparación con otras economías desarrolladas.

En este sentido, no es posible realizar un muestreo representativo, ya que por diseño del modelo se debe tomar como dados los componentes del Índice General de la Bolsa de Valores de Lima.

Las variables económicas agregadas a utilizar son:

Consumo agregado

Exportaciones

Inversión agregada

Tipo de cambio real

Como expresamos anteriormente nuestra propuesta se basa en Leigh (1997), por ende, nuestra propuesta se basa también en la idea planteada por Leigh: Analizar como la bolsa de valores juega un rol en el desarrollo de los países. De la misma manera como el autor aplica este análisis a la bolsa de valores de Singapur, nuestra propuesta es analizarlo para el caso del Perú, pues como se sabe en los últimos años hemos ido teniendo un crecimiento económico sostenido, solo con atípicos quiebres de caídas de orden de $2 \%$.

Para Leigh el buen funcionamiento de las bolsas de valores en los diversos países tiene un grado positivo de afectación a las economías sobre todo en el crecimiento del ahorro, mejor localización de las inversiones y mejor utilización de los recursos de las diversas empresas; es de esperar que las bondades de la bolsa de valores y en general del sistema financiero en todo el mundo agilice en gran medida estos efectos esperados.

Leigh (1997) indica que el papel de bolsa eficiente en el sentido discutido anteriormente, como: 
"El énfasis es que tener un mercado de valores en desarrollo mejora la eficiencia de la inversión. Un mercado de valores que funcione bien puede realizar sus funciones de asignación a través de la fijación de precios de la acción." (Leigh 1997: p. 5).

Luego nos explica dos conceptos de eficiencia de los precios de las acciones los cuales son: la evaluación fundamental y la información de arbitraje. Esta evidencia muestra cómo se explicó al principio del documento la eficiencia del mercado, pues el mercado de valores muestra de manera eficiente el precio de las acciones. También hay una discusión necesaria sobre las nuevas tecnologías incorporadas a la bolsa y algunas innovaciones en los instrumentos financieros.

Se muestra así con alguna literatura la evidencia que el mercado de valores es eficiente, mostrando algunas reseñas sobre la bolsa de Londres o la de New York. Ahora bien, lo que el autor profundizara será la eficiencia en la bolsa de valores de Singapur, los cuales serán relacionados con las algunas variables macroeconómicas, por ende, pasara primero a discutir los principales componentes de la bolsa de valores de Singapur.

Obteniendo los datos de los precios de las acciones se puede hacer la prueba empírica para obtener los resultados de la eficiencia del mercado de valores. Primero se analiza la forma débil de la eficiencia de la bolsa de valores; para esto usa el modelo de camino aleatorio que ya se especificó anteriormente:

$$
s r_{t}=\frac{p_{t+1}-p_{t}+d_{t}}{p_{t}}
$$

Donde $p_{t}$ el precio por acción, $d_{t}$ el dividendo pagado a tiempo y $s r_{t}$ es el retorno nominal de la acción.

Luego en base a los datos y a la recolección de la literatura que se obtuvo en otros periodos se realiza una la regresión de series de tiempo pertinente; usando un test de raíz unitaria basado en los argumentos de Dickey-Fuller. También analiza algunos test de cointegración analizando el Durbin-Watson. Finalmente compara con el test de Phillips-Perron.

De todas estas pruebas se puede colegir: 
"Estos resultados sugieren que los rendimientos de las acciones son estacionarios $\mathrm{y}$, por lo tanto, el mercado es eficiente en el sentido débil, ya que los cambios sucesivos en los precios son independientes e impredecibles." (Leigh 1997: p. 7).

Entonces para probar esta hipótesis de eficiencia semi-fuerte se usará el método de series de tiempo VAR. Este método tiene la ventaja de capturar el análisis tanto de corto como de largo plazo ${ }^{3}$. Luego también los test para medir la integración.

Es evidente que la literatura al respecto es abundante, el autor cita un trabajo donde relaciona la demanda agrega a la eficiencia de la bolsa de valores, pero Leigh, propone un esquema más rico de análisis, donde se ponen más variables para obtener una visión más completa de la hipótesis de eficiencia, por tanto, las tres relaciones mostradas a continuación nos muestran las cómo se comportan las variables, estas ecuaciones con:

$$
\left[\begin{array}{c}
(s r-\pi)_{t} \\
c_{t} \\
i_{t} \\
x_{t} \\
\operatorname{rer}_{t}
\end{array}\right]=\left[\begin{array}{llllll}
a_{11} & a_{12} & a_{13} & a_{14} & a_{15} & a_{16} \\
a_{21} & a_{22} & a_{23} & a_{24} & a_{25} & a_{26} \\
a_{31} & a_{32} & a_{33} & a_{34} & a_{35} & a_{36} \\
a_{41} & a_{42} & a_{43} & a_{44} & a_{45} & a_{46} \\
a_{51} & a_{52} & a_{53} & a_{54} & a_{55} & a_{56}
\end{array}\right]\left[\begin{array}{c}
(s r-\pi)_{t-1} \\
c_{t-i} \\
i_{t-i} \\
x_{t-i} \\
r e r_{t-i}
\end{array}\right] \ldots
$$

Luego las variables para esta ecuación (1) son: $(s r-\pi)$ es el retorno de la bolsa de valores, descontando la inflación, $c$ es el consumo, $i$ es la inversión y $x$ son las exportaciones (todas en términos reales) y rer es la tasa de intercambio real. Esta ecuación está relacionada con los componentes de la demanda agregada.

Luego con los datos que obtiene hace primero un test de cointegración de Johansen, entonces cuando se aplica el test muestra que en el largo plazo puede dar la forma semi-fuerte de eficiencia. Finalmente hace varios test asintóticos y sus implicancias de eficiencia como lo cual se puede concluir:

"El rechazo de la hipótesis nula de la ineficiencia del mercado de valores y la aceptación de la alternativa tanto en el sistema de demanda agregada como en el de función de producción agregada es un resultado significativo." (Leigh 1997: p. 21). 
Para el autor se concluye que la bolsa de valores de Singapur es eficiente en sentido semi-fuerte. Para nuestro estudio, nos inspiramos en estos contrastes que realizo Leigh pero para el caso del Perú por tanto la siguiente parte nos centraremos en hacer un modelo empírico que trate de demostrar estas relaciones.

\section{Resultados y Discusión}

Tenemos el modelo de donde se analiza el efecto de las variables macroeconómicas a la bolsa de valores, este modelo se puede especificar como el modelo VAR señalado.

Con datos de 1990 al 2018 expresado en trimestres, hallamos el VAR sin restringir y apoyándonos en el criterio de selección del número de rezagos, calculamos el modelo en la Tabla 1.

\section{Tabla 1}

Selección de rezagos del modelo

VAR Lag Order Selection Criteria

Endogenous variables: SR_PI CONSUM INVER EXPOR RER

Exogenous variables: C

Date: 03/20/19 Time: 15:09

Sample: 1117

Included observations: 99

\begin{tabular}{cllllll}
\hline Lag & LogL & LR & FPE & AIC & SC & HQ \\
\hline 0 & -4188.486 & NA & $4.26 \mathrm{e}+30$ & 84.71689 & 84.84796 & 84.76992 \\
1 & -3721.405 & 877.5466 & $5.64 \mathrm{e}+26$ & 75.78596 & 76.57236 & 76.10414 \\
2 & -3635.352 & 152.9825 & $1.65 \mathrm{e}+26$ & 74.55257 & 75.99431 & 75.13590 \\
3 & -3567.134 & 114.3872 & $6.96 \mathrm{e}+25$ & 73.67947 & 75.77653 & 74.52794 \\
4 & -3487.741 & 125.1029 & $2.36 \mathrm{e}+25$ & 72.58063 & $75.33303^{*}$ & $73.69426^{*}$ \\
5 & -3452.877 & 51.41658 & $1.99 \mathrm{e}+25$ & 72.38135 & 75.78908 & 73.76012 \\
6 & -3422.432 & $41.82269 *$ & $1.87 \mathrm{e}+25^{*}$ & 72.27136 & 76.33442 & 73.91528 \\
7 & -3396.054 & 33.57166 & $1.94 \mathrm{e}+25$ & $72.24352^{*}$ & 76.96192 & 74.15260 \\
8 & -3375.476 & 24.11153 & $2.32 \mathrm{e}+25$ & 72.33286 & 77.70659 & 74.50708 \\
\hline
\end{tabular}

* indicates lag order selected by the criterion

LR: sequential modified LR test statistic (each test at 5\% level)

FPE: Final prediction error

AIC: Akaike information criterion

SC: Schwarz information criterion

HQ: Hannan-Quinn information criterion 
Luego como ya tenemos el número de rezagos óptimos ya podemos reformular el modelo VAR, de donde nos interesa ver las funciones de impulso respuesta para ver las relaciones en el largo plazo, los cuales se aprecian en la Figura 1.

\section{Figura 1}

Respuesta de las variables del modelo a 1 innovación de las otras variables

Response to Cholesky One S.D. (d.f. adjusted) Innovations
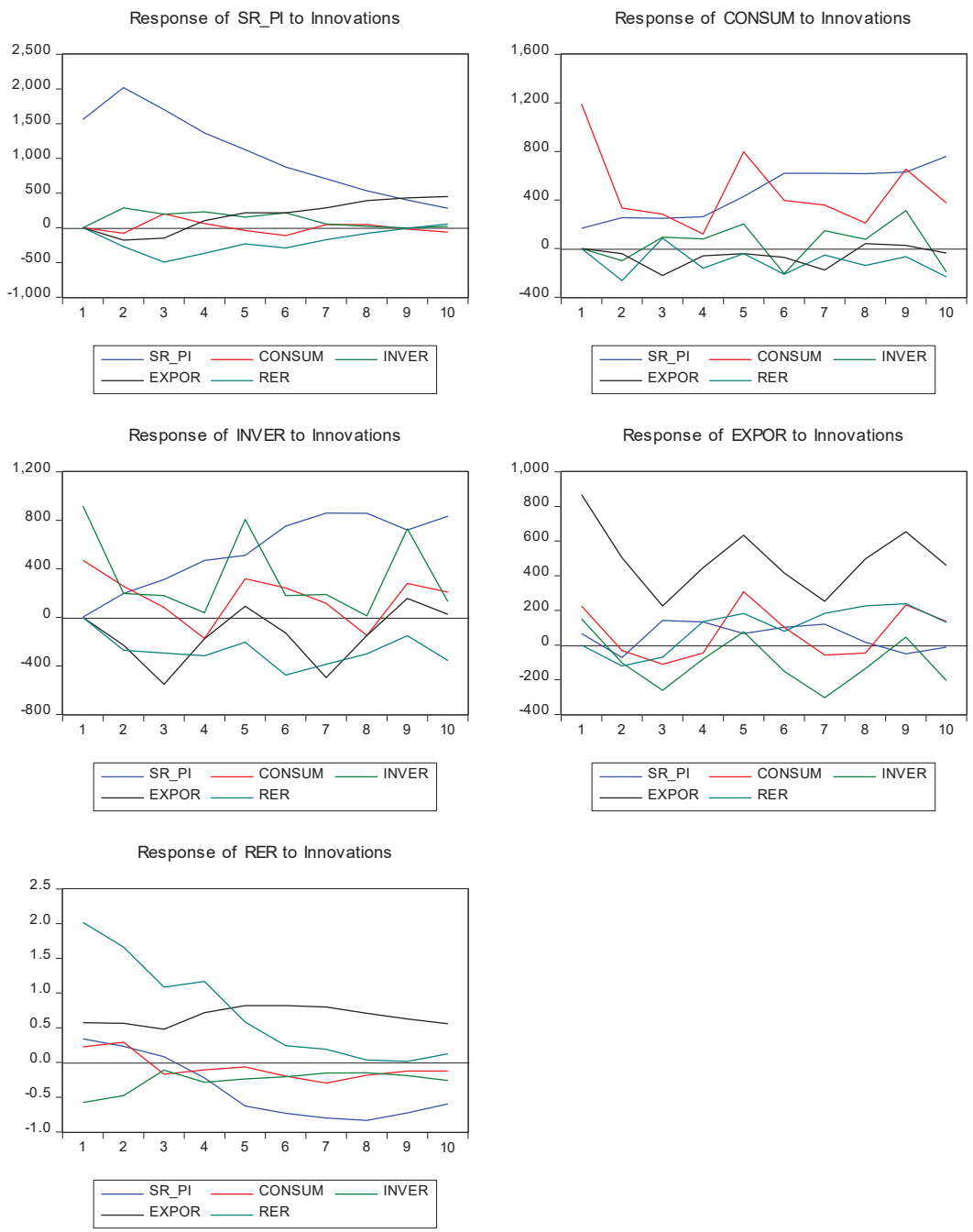
Como se puede ver, para nuestro caso postulamos que la variable que queremos explicar es el índice de la bolsa, por ende, presentamos los impulsos respuestas solo para ese caso (ver Figura 2)

Figura 2

Respuesta de $\left(s_{r}-r_{f}\right)$ a innovaciones en las variables del modelo

Response of SR_PI to Innovations

using Cholesky (d.f. adjusted) Factors

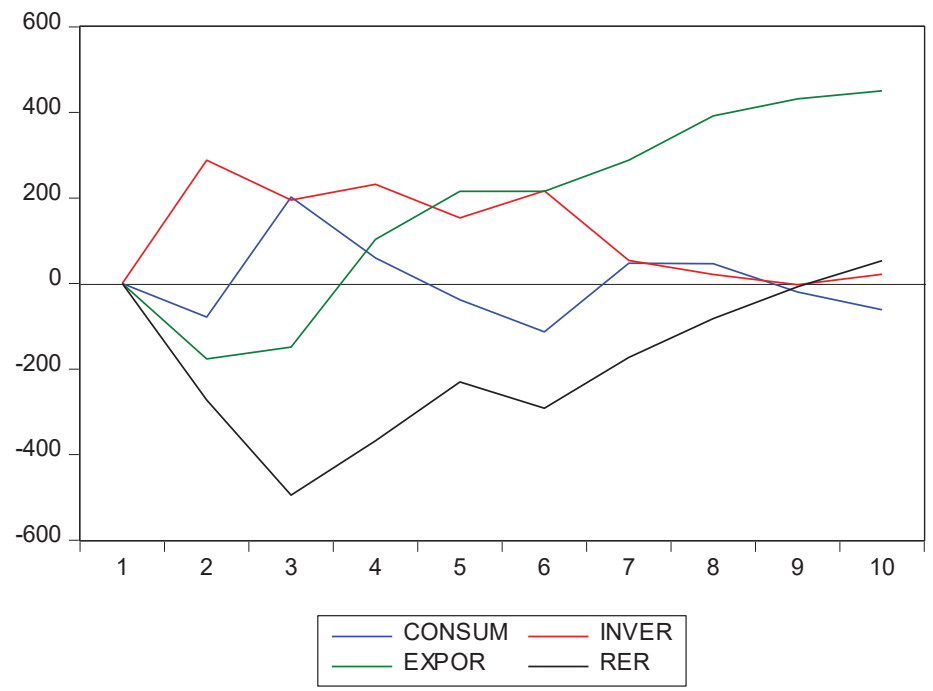

Se debe tener en cuenta que las relaciones de impulso respuesta dan una explicación a largo plazo, estas tendencias son a largo plazo para cada variable. Como vemos en estos cuadros se resumen todos los posibles choques a largo plazo de las variables, también podemos ver el VAR en sus resultados estadísticos tal como la Tabla 2.

Como podemos ver los estadísticos que obtenemos nos resultan significativos, el R cuadrado que obtenemos nos salen altos, también podemos ver que el F-static también es significativo. Los resultados de los test de Traza y de cointegración son coherentes con los del modelo estimado. 
Pensamiento Crítico Vol. 26. $\mathrm{N}^{\circ} 2$

Tabla 2

Estimación del modelo VAR con los rezagos óptimos estimados (se presentan los coeficientes del primer rezago).

Vector Autoregression Estimates

Date: 03/20/19 Time: 15:15

Sample (adjusted): 13115

Included observations: 103 after adjustments

Standard errors in () \& t-statistics in []

\begin{tabular}{|c|c|c|c|c|c|}
\hline & SR_PI & CONSUM & INVER & EXPOR & RER \\
\hline \multirow[t]{3}{*}{ SR_PI(-1) } & 1.341214 & 0.149724 & 0.139635 & -0.055547 & $-4.39 \mathrm{E}-05$ \\
\hline & (0.10969) & $(0.08442)$ & $(0.07243)$ & $(0.06403)$ & $(0.00016)$ \\
\hline & [12.2270] & [1.77348] & [ 1.92799] & {$[-0.86745]$} & {$[-0.28264]$} \\
\hline \multirow[t]{3}{*}{ CONSUM(-1) } & -0.117236 & 0.376073 & 0.211004 & -0.032692 & $7.95 \mathrm{E}-05$ \\
\hline & $(0.13434)$ & (0.10339) & $(0.08870)$ & $(0.07842)$ & (0.00019) \\
\hline & {$[-0.87267]$} & [3.63726] & [2.37885] & {$[-0.41686]$} & [0.41772] \\
\hline \multirow[t]{3}{*}{ INVER(-1) } & 0.248135 & -0.198065 & 0.158278 & -0.250920 & $-2.13 \mathrm{E}-05$ \\
\hline & $(0.14002)$ & $(0.10776)$ & $(0.09245)$ & $(0.08174)$ & $(0.00020)$ \\
\hline & [ 1.77220$]$ & {$[-1.83800]$} & [1.71211] & [-3.06991] & {$[-0.10719]$} \\
\hline \multirow[t]{3}{*}{ EXPOR(-1) } & -0.114196 & 0.038758 & -0.174328 & 0.620529 & 0.000107 \\
\hline & $(0.20609)$ & $(0.15862)$ & $(0.13607)$ & $(0.12031)$ & (0.00029) \\
\hline & {$[-0.55410]$} & [ 0.24435] & [-1.28114] & [5.15783] & {$[0.36755]$} \\
\hline \multirow[t]{3}{*}{$\operatorname{RER}(-1)$} & -134.9521 & -130.9613 & -134.6127 & -59.54328 & 0.824684 \\
\hline & (79.0563) & $(60.8447)$ & (52.1973) & (46.1499) & (0.11199) \\
\hline & {$[-1.70704]$} & [-2.15239] & [-2.57892] & {$[-1.29022]$} & [7.36382] \\
\hline \multirow[t]{3}{*}{$\mathrm{C}$} & 2877.974 & 1750.285 & 12547.91 & -1316.136 & 35.83124 \\
\hline & $(6412.10)$ & (4934.99) & $(4233.62)$ & $(3743.12)$ & (9.08339) \\
\hline & [0.44883] & {$[0.35467]$} & [2.96388] & {$[-0.35161]$} & [3.94470] \\
\hline R-squared & 0.967491 & 0.997439 & 0.990062 & 0.991786 & 0.819388 \\
\hline Adj. R-squared & 0.959562 & 0.996814 & 0.987638 & 0.989783 & 0.775336 \\
\hline Sum sq. resids & $2.00 \mathrm{E}+08$ & $1.18 \mathrm{E}+08$ & 87114407 & 68098082 & 401.0171 \\
\hline S.E. equation & 1561.086 & 1201.470 & 1030.714 & 911.2982 & 2.211437 \\
\hline F-statistic & 122.0182 & 1596.601 & 408.4533 & 495.0698 & 18.60057 \\
\hline Log likelihood & -891.7813 & -864.8122 & -849.0229 & -836.3398 & -216.1533 \\
\hline Akaike AIC & 17.72391 & 17.20024 & 16.89365 & 16.64737 & 4.604919 \\
\hline Schwarz SC & 18.26109 & 17.73741 & 17.43083 & 17.18455 & 5.142097 \\
\hline Mean dependent & 8831.603 & 59450.64 & 17538.09 & 21288.07 & 97.75069 \\
\hline S.D. dependent & 7763.018 & 21285.46 & 9270.284 & 9015.703 & 4.665604 \\
\hline \multicolumn{2}{|c|}{ Determinant resid covariance (dof adj.) } & $8.87 \mathrm{E}+24$ & & & \\
\hline \multicolumn{2}{|c|}{ Determinant resid covariance } & $2.84 \mathrm{E}+24$ & & & \\
\hline \multicolumn{2}{|l|}{ Log likelihood } & -3630.453 & & & \\
\hline \multicolumn{2}{|c|}{ Akaike information criterion } & 72.53307 & & & \\
\hline \multicolumn{2}{|l|}{ Schwarz criterion } & 75.21896 & & & \\
\hline \multicolumn{2}{|c|}{ Number of coefficients } & 105 & & & \\
\hline
\end{tabular}


Leigh indica que: "Sin embargo, el análisis de los resultados de cointegración que se muestran arriba solo se puede considerar como una prueba parcial para la eficiencia semi-fuerte a largo plazo. Para implementar una prueba de sistemas completa, debemos realizar una prueba de LR e imponer restricciones en la matriz de estimación $\beta$ ', en la cual la hipótesis nula es una ineficiencia del mercado de valores semi fuerte. Para probar la validez de esta hipótesis en los tres sistemas analizados aquí, podemos imponer una restricción de la forma,

$H_{0}: \beta=C \phi$

Para nuestro caso tenemos que la matriz de restricción será:

$$
\left(\begin{array}{cccc}
1 & 0 & 0 & 0 \\
0.3 & 0 & 0 & 0 \\
-0.7 & 1 & 0 & 0 \\
-0.6 & 0 & 1 & 0 \\
42.8 & 0 & 0 & 1
\end{array}\right)
$$

Luego hallamos estas restricciones para nuestro modelo y obtenemos:

\section{Tabla 3}

Restricciones aplicadas al modelo

\begin{tabular}{|c|c|c|c|c|}
\hline \multicolumn{5}{|l|}{ Restrictions: } \\
\hline \multicolumn{5}{|c|}{$\mathrm{B}(1,1)=1, \mathrm{~B}(1,2)=0.3, \mathrm{~B}(1,3)=-0.7, \mathrm{~B}(1,4)=-0.6, \mathrm{~B}(1,5)=42.8, \mathrm{~A}(2,1)=0, \mathrm{~A}(3,1)=0, \mathrm{~A}(4,1)=0, \mathrm{~A}(5,1)=0$} \\
\hline \multicolumn{5}{|c|}{ Tests of cointegration restrictions: } \\
\hline Hypothesized & Restricted & LR & Degrees of & \\
\hline No. of CE(s) & Log-likehood & Statistic & Freedom & Probability \\
\hline 1 & -3613.413 & 25.50658 & 8 & 0.001275 \\
\hline 2 & -3593.035 & 9.622101 & 6 & 0.141495 \\
\hline 3 & -3583.766 & 3.136605 & 4 & 0.535231 \\
\hline 4 & -3580.304 & 0.490844 & 2 & 0.782374 \\
\hline \multicolumn{5}{|c|}{1 Cointegrating Equation(s): Convergence achieved after 4 iterations. } \\
\hline \multicolumn{5}{|c|}{ Restricted cointegrating coefficients (standard error in parentheses) } \\
\hline SR_PI & CONSUM & INVER & EXPOR & RER \\
\hline 1.000000 & 0.300000 & -0.700000 & -0.600000 & 42.80000 \\
\hline$(0.00000)$ & $(0.00000)$ & $(0.00000)$ & $(0.00000)$ & $(0.00000)$ \\
\hline \multicolumn{5}{|c|}{ Adjustment coefficients (standard error in parentheses) } \\
\hline \multirow[t]{2}{*}{ D(SR_PI) } & -0.199669 & & & \\
\hline & $(0.06352)$ & & & \\
\hline D(CONSUM) & 0.000000 & & & \\
\hline
\end{tabular}


Tabla 3: Continuación...

\begin{tabular}{|c|c|c|c|c|}
\hline \multirow{3}{*}{ D(INVER) } & \multicolumn{4}{|l|}{$(0.00000)$} \\
\hline & \multicolumn{4}{|l|}{0.000000} \\
\hline & \multicolumn{4}{|l|}{$(0.00000)$} \\
\hline \multirow[t]{2}{*}{ D(EXPOR) } & \multicolumn{4}{|l|}{0.000000} \\
\hline & \multicolumn{4}{|l|}{$(0.00000)$} \\
\hline \multirow[t]{2}{*}{ D(RER) } & \multicolumn{4}{|l|}{0.000000} \\
\hline & \multicolumn{4}{|l|}{$(0.00000)$} \\
\hline \multicolumn{5}{|c|}{2 Cointegrating Equation(s): Convergence achieved after 28 iterations. } \\
\hline \multicolumn{5}{|c|}{ Restricted cointegrating coefficients (not all coefficients are identified) } \\
\hline SR_PI & CONSUM & INVER & EXPOR & RER \\
\hline 1.000000 & 0.300000 & -0.700000 & -0.600000 & 42.80000 \\
\hline-0.000237 & $-8.69 \mathrm{E}-05$ & 0.000222 & $7.27 \mathrm{E}-05$ & -0.012351 \\
\hline \multicolumn{5}{|c|}{ Adjustment coefficients (standard error in parentheses) } \\
\hline \multirow[t]{2}{*}{ D(SR_PI) } & -0.507333 & -904.4180 & & \\
\hline & $(0.12697)$ & $(269.171)$ & & \\
\hline \multirow[t]{2}{*}{ D(CONSUM) } & 0.000000 & -422.9050 & & \\
\hline & $(0.00000)$ & (95.4461) & & \\
\hline \multirow[t]{2}{*}{ D(INVER) } & 0.000000 & -85.04124 & & \\
\hline & $(0.00000)$ & (84.7624) & & \\
\hline \multirow[t]{2}{*}{ D(EXPOR) } & 0.000000 & -279.4093 & & \\
\hline & $(0.00000)$ & (77.9363) & & \\
\hline \multirow[t]{2}{*}{ D(RER) } & 0.000000 & -0.294608 & & \\
\hline & $(0.00000)$ & $(0.20379)$ & & \\
\hline
\end{tabular}

Como se puede ver el LR test es alto en todos los casos, excepto en la primer, lo que nos quiere reflejar esto es similar a la cointegración en al menos 1 es significativamente débil, pero para nuestro caso de las otras relaciones de cointegración decimos que si son significativas, para esto basta ver la probabilidad que pasan del 5\%, que por ende se rechaza la hipótesis nula que el mercado es ineficiente en sentido semi-fuerte, por ende estas restricciones nos muestran un apoyo a la hipótesis de eficiencia en el largo plazo sobre para el mercado peruano.

Toda esta información se puede resumir en la siguiente matriz, siguiendo a Leigh (1995):

Test de Johansen con cinco rezagos y una constante. 


$$
\left(\begin{array}{cccccc}
\text { Test } & \boldsymbol{\rho}=\mathbf{0} & \boldsymbol{\rho} \leq \mathbf{1} & \boldsymbol{\rho} \leq \mathbf{2} & \boldsymbol{\rho} \leq \mathbf{3} & \boldsymbol{\rho} \leq \mathbf{4} \\
\mu_{i} & 0.288998 & 0.216396 & 0.111451 & 0.041063 & 0.001376 \\
\text { Trace } & 76.13314 & 41.34293 & 16.47007 & 4.417242 & 0.140413 \\
5 \% C V & 60.06141 & 40.17493 & 24.27596 & 12.32090 & 4.129906 \\
\mu_{\max } & 34.79022 & 24.87285 & 12.05283 & 4.276829 & 0.140413 \\
5 \% C V & 30.43961 & 24.15921 & 17.79730 & 11.22480 & 4.129906
\end{array}\right)
$$

También podemos tener la matriz $\beta$ transpuesta que es las relaciones de cointegración normalizadas, note que la traza es unitaria pues el significado que se le da a cada ecuación es una variable de correlación con las otras variables. Esta matriz es:

La matriz propia, $\beta^{T}$

$$
\left(\begin{array}{cccccc}
\beta^{T} & s r-\pi & c & i & x & \text { rer } \\
1 & 1.000 & 0.338 & -0.737 & -0.616 & 42.84 \\
2 & 2.957 & 1.000 & -2.181 & -1.823 & 126.73 \\
3 & -1.355 & -0.458 & 1.000 & 0.836 & -58.09 \\
4 & -1.621 & -0.548 & 1.195 & 1.000 & -69.48 \\
5 & 0.023 & 0.0078 & -0.017 & -0.0143 & 1.000
\end{array}\right)
$$

A partir de esta matriz propia podemos obtener la ecuación a largo plazo de la influencia de las variables macroeconómicas en la bolsa de valores, por ende, podemos tener la ecuación de influencias a largo plazo el cual se puede expresar como:

$$
(s r-\pi)=-0.338 * c+0.737 * i+0.616 * x-42.84 * r e r
$$

Esta ecuación se puede interpretar como una correlación, se puede decir que el largo plazo hay una relación negativa del índice de desarrollo de la bolsa de valores con el consumo, positiva con la inversión, positiva con la exportación e inversa con el tipo de cambio. Estas relaciones resultan coherentes con la teoría económica por ende nuestra hipótesis queda sustentada.

\section{Conclusiones}

En el presente trabajo nos propusimos estimar un modelo en la metodología de series de tiempo, podemos concluir que se verifica una relación entre el índice de la bolsa de valores y algunas variables macro denominadas fundamentales en la práctica reciente, este modelo, nos permita estimar si el mercado de valores nacional presenta eficiencia en el sentido semi-fuerte. 
Con base en los resultados obtenidos en la presente investigación, se llegó a las siguientes conclusiones:

El mercado de valores muestra eficiencia en el sentido semi-fuerte respecto a las variables conocidas como fundamentos económicos, tales como consumo agregado, inversión, exportaciones y el tipo de cambio real, tanto en los efectos temporales como respecto a los efectos acumulados los resultados están en línea con los efectos esperados.

El marco metodológico utilizado nos permitió analizar mediante técnicas de series de tiempo adaptadas el comportamiento de la bolsa de valores y su relación con los fundamentos económicos tradicionales.

Estos resultados están en línea con Iqbal et al.(2017), de manera general con Poterba y Summers (1989) y Karki (2018), adicionalmente nuestro modelo nos permite testear la hipótesis de eficiencia semi fuerte, obteniendo n línea tambien con Leigh (2007) que el mercado nacional presenta este tipo de eficiencia.

Tanto para los gestores de portafolio profesionales como para los inversores nacionales es importante saber si el mercado es eficiente (por lo menos en el sentido semi fuerte), tanto para la gestión del riesgo como para la determinación de los objetivos de inversión. Nuevos modelos podrían explorar esta característica a nivel de sectores económicos como incluso de carteras de inversionistas institucionales (AFPs y fondos mutuos) que invierten en el mercado peruano.

El planteamiento teórico que hemos utilizado y el modelo econométrico de series de tiempo usadas, utilizadas en varios mercados emergentes nos podrían permitir realizar un análisis comparativo para ampliar el conocimiento sobre la dinámica de las bolsas de valores en estos mercados, por lo que su aplicación en otras economías emergentes se puede explorar con el modelo utilizado.

\section{Referencias Bibliográficas}

Bolsa de Valores de Lima. Anuario estadístico. Varios números.

Campbell, J., Lo, A.W. \& MacKinlay, A.C. (1997). The econometric analysis of financial markets. New Jersey: Princeton University Press. 
De Gregorio, J., \& Guidotti, P. E. (1995). Financial Development and Economic Growth. World Development, 23(3), 433-448.

De la Torre, A.; Gozzi, J.C.; Schmukler, S (2007) Financial Development: Maturing and Emerging Policy Issues. The World Bank Research Observer, Volume 22, Issue 1, Spring 2007: pp. 67-102, Disponible en SSRN: https://ssrn.com/abstract $=2172536$

Demirgüç-Kunt, A., \& Levine, R. (1993). Stock Market Development and Financial Intermediary Growth. World Bank Policy Research Working Paper 1159.

Fama, E. F. (1970). Efficient Capital Markets: A Review Of Theory And Empirical Work. The Journal of Finance, 25(2), 383-417.

Fama, E. F. (1991). Efficient Capital Markets: II. The Journal of Finance, 46(5), 1575-1617.

Fama, E. F. (1998). Market efficiency, long-term returns, and behavioral finance. Journal of Financial Economics, 49 (1), 283-306.

Greene W.H. (1999). Análisis Econométrico. Prentice Hall Iberia, 3ạ Edición.

Iqbal, P., Nawaz, S., Umer, Z. (2017). Impact of macroeconomic fundamentals on stock exchange market: Empirical evidence from Pakistan. Paradigms: $A R e-$ search Journal of Commerce, Economics, and Social Sciences. 11, No. 1, 103110. DOI: $10.24312 /$ paradigms 110116

Karki, D. (2018). Stock Market Responses to Macroeconomic Dynamics: Testing for Long-Run Equilibrium in Nepal. Pravaha Journal. Vol. 24 No. 1. pp64-82. DOI: https://doi.org/10.3126/pravaha.v24i1.20227

La Porta, R., Lakonishok, J, Shleifer, A. y Vishny, R. (1997). Good News for Value Stocks: Further Evidence on Market Efficiency. Journal of Finance, Vol. 52 No. 2, pp. 859-874.

Leigh, L. (1997). Stock Return Equilibrium and Macroeconomic Fundamentals. International Monetary Fund Working Paper: No. 97/15.

Mongrut-Montalván, S. (2011). Market efficiency: An empirical survey in Peru and other selected countries. Apuntes 51, Universidad del Pacífico.

Ojah, K., \& Karemera, D. (1999). Random Walks and Market Efficiency Tests of Latin American Emerging Equity Markets: A Revisit. Financial Review, $34(2), 57-72$.

Poterba, J. y Summers, (1989). Mean reversion in stock prices: Evidence and Implications. Journal of Financial Economics, Vol 22, No. 1, pp. 27-59. 
Stock, J. y Watson, M. (2012). Introducción a la Econometría. 3ae ed. Madrid: Pearson Educación.

Summers, L.H. (1986). Does the stock market rationally reflect fundamental value? Journal of Finance, Vol 41, pp. 591-601.

Wooldridge, J. (2010). Introducción a la Econometría. 4⿳ạ ed. México, D.F.: Cengage Learning.

\section{Notas al final}

1 Este artículo es de investigación científica. El presente escrito resume el trabajo de investigación para optar el Grado de Magíster en Economía con mención en Finanzas por la Universidad Nacional Mayor de San Marcos denominado: "Estimación de la Eficiencia en la Bolsa de Valores de Lima (1990-2018) mediante sus fundamentos económicos".

2 Economista, Universidad Nacional Federico Villarreal, Lima, Perú. Egresado de Maestría en Finanzas, Universidad Nacional Mayor de San Marcos. Egresado de Maestría en Gestión Pública, Universidad San Martin de Porras. Egresado de Doctorado en Economía, Universidad Inca Garcilaso de la Vega. Oficial de Aduanas, Escuela Nacional de Aduanas, SUNAT. Correo: dchavezv@sunat,gob.pe

3 Para tener una visión más clara de este tema se puede revisar: Campbell y MacKinla A.C. (1997), Campbell y Shiller (1987), Campbell y Yogo, M. (2003). Esta referencia se puede ver en la bibliografía. 
